\title{
Some characterizations of inner product spaces based on angle
}

\author{
S.M.S. Nabavi Sales \\ Department of Mathematics and Computer Sciences, Hakim Sabzevari University, P.O. Box 397, \\ Sabzevar, Iran
}

\begin{abstract}
A problem in functional analysis that arises naturally is about finding necessary and sufficient conditions for a normed space to be an inner product space. By answering this question, mathematicians try to understand the inner product and normed spaces features. In this note, we have discussed this issue and we prove some results concerned with it. We introduce a notion of angle between two vectors in a normed space, denoted by $A_{\theta}(.,$. where $\theta \neq \frac{k \pi}{2}$. We also speak about a notion of orthogonality concerning it, we call it $\theta$-orthogonality.
\end{abstract}

Mathematics Subject Classification (2010). 46C15

Keywords. inner product, normed space, angle, orthogonality

\section{Introduction}

We know that every inner product space is a normed space but the converse is not true in general. We very soon realize that finding the conditions on normed spaces under which such spaces become inner product spaces is interesting, because of the significant natural geometric properties of the Hilbert spaces which fail in general normed spaces. In fact, this issue was first raised by Frećhet [7] in 1935, who gave a characterization of inner product spaces based on geometrical aspects of underlying spaces. Along with his work, Jordan and von Neumann [10] discovered that inner product spaces are precisely those normed spaces which satisfy the parallelogram law. In 1947 Day presented an improvement of the result of Jordan and von Neumann. In fact he proved that a normed space $X$ is an inner product space if and only if

$$
\|x+y\|^{2}+\|x-y\|^{2}=4
$$

for every $x$ and $y$ with norm one in $X$; see [3]. Since then the problem was considered by many authors who investigated some types of orthogonality and explored the geometrical aspects of underlying spaces. These studies led to the discovery of many important characterizations. Only Amir expresses itself 350 samples of these characterizations in his book, see [1]. Some investigations in this field carried out by Diminnie, Andalafte and Freese in the 80s who introduced some types of orthogonality and a concept of angle in

Email addresses: sadegh.nabavi@gmail.com; sadegh.nabavi@hsu.ac.ir Received: 31.10.2016; Accepted: 18.01.2017 
normed spaces; $[5,6]$. For example, among other things, they introduced a notion of angle $A(x, y)$ between two vectors $x$ and $y$ in normed space $\mathscr{X}$ as follows:

$$
A(x, y):=\cos ^{-1}\left[\frac{2-\left\|\frac{x}{\|x\|}-\frac{y}{\|y\|}\right\|^{2}}{2}\right] .
$$

Based on this concept, they could obtain some characterizations of inner product spaces. Concerned with the concept of angle they consider a type of orthogonality in normed space and investigated some relations between this type of orthogonality and the others which had been introduced by James in $[8,9]$. From this way they could achieve some other characterizations of inner product spaces as well.

It should be mentioned that dimensionality plays no role in many characterizations. This is the case by virtue the well-known fact that a normed space is an inner product space if and only if so is every two dimensional subspace of it. However in some characterizations it is essential. For instance the symmetry of Birkhoff-James orthogonality characterizes inner product spaces when the dimensional of the space is three or more than three; see[3,8].

In this paper we introduce a notion of angle between two elements of a normed space, denoted by $A_{\theta}(.,$.$) where \theta \neq \frac{k \pi}{2}$, which in the particular case $\theta=\frac{\pi}{4}$ coincides with the angle mentioned above, and based on it we present some new characterizations of inner product spaces. One can observe some analogies between these notions and the concept of $p$-angular distance and compare the related characterizations; see [2]. The spaces in this paper are all assumed to be real and $\mathscr{X}$ is often used to indicate a normed space.

\section{Main results}

First we see the following lemmata of [3] which we need in the sequel.

Lemma 2.1. Let $\mathscr{X}$ be a two dimensional real normed space and $S$ be the set of points of norm one. Then $S$ is an ellipse if and only if $\mathscr{X}$ is an inner product space.

Lemma 2.2. Let $\mathscr{X}$ be a two dimensional real normed space. Then:

i) The minimal ellipse circumscribed about a symmetric closed convex $S$ touches $S$ in at least four points;

ii) The minimal ellipse inscribed a symmetric closed convex $S$ touches $S$ in at least four points.

Now we could present the first our main result. In fact it is a slight modification of the well-known theorem of Day; see [3, theorem 2.1] and [4].

Theorem 2.3. A normed space $\mathscr{X}$ is an inner product space if and only if it satisfies the following requirement

$$
\|\sin \theta x+\cos \theta y\|^{2}+\|\sin \theta x-\cos \theta y\|^{2} \sim 2
$$

for some $\theta \neq \frac{k \pi}{2}$ and every $x, y \in \mathscr{X}$ with $\|x\|=\|y\|=1$ which $\sim$ stands for any one of the relations $=, \geq$, or $\leq$.

Proof. A routine computations shows that in any inner product space the conditions (2.1) are held. Thus we show that, from (2.1) we could conclude that $\mathscr{X}$ is an inner product space. To this aim, it is sufficient proving it for an arbitrary two dimensional subspace of $\mathscr{X}$. Without loss of generality we may assume that $\theta \in\left(0, \frac{\pi}{2}\right)$. Let $\mathscr{P}$ be a two dimensional subspace of $\mathscr{X}$ and let $S$ be the set of points of norm 1 in $\mathscr{P}$. By Lemma 2.1 suffice it proving that $S$ is an ellipse. First we assume that $\sim$ is $\geq$. Let $S^{\prime}$ be the ellipse of maximum area inside $S$. We want to show that $S$ coincides $S^{\prime}$. For, let $A$ be the set of intersection of $S$ and $S^{\prime}$. Thus $A$ is closed. By Lemma 2.2, $A$ is contained at least 
four points. Define a new norm $\|\cdot\|^{\prime}$ in $\mathscr{P}$ which $S^{\prime}$ is the unit sphere. Since $S^{\prime}$ is inside $S$ thus $\|x\| \leq\|x\|^{\prime}$ for every $x \in \mathscr{P}$. Now, let $x$ and $y \neq \pm x$ be two points of $A$ which are end points of an open arc of the complement of $A$. $\left(\mathscr{P},\|.\|^{\prime}\right)$ is inner product space by Lemma 2.1. Thus

$$
\|\sin \theta x+\cos \theta y\|^{2}+\|\sin \theta x-\cos \theta y\|^{\prime 2}=2 .
$$

Hence

$$
\begin{aligned}
2 & =\|\sin \theta x+\cos \theta y\|^{\prime 2}+\|\sin \theta x-\cos \theta y\|^{\prime 2} \\
& \geq\|\sin \theta x+\cos \theta y\|^{2}+\|\sin \theta x-\cos \theta y\|^{2} \geq 2
\end{aligned}
$$

which implies that

$\left(\|\sin \theta x+\cos \theta y\|^{\prime 2}-\|\sin \theta x+\cos \theta y\|^{2}\right)+\left(\|\sin \theta x-\cos \theta y\|^{\prime 2}-\|\sin \theta x-\cos \theta y\|^{2}\right)=0$. Since $\|\sin \theta x+\cos \theta y\|^{\prime 2}-\|\sin \theta x+\cos \theta y\|^{2}$ and $\|\sin \theta x-\cos \theta y\|^{\prime 2}-\|\sin \theta x-\cos \theta y\|^{2}$ are positive hence

$$
\|\sin \theta x+\cos \theta y\|^{\prime}=\|\sin \theta x+\cos \theta y\|
$$

and

$$
\|\sin \theta x-\cos \theta y\|^{\prime}=\|\sin \theta x-\cos \theta y\| .
$$

Since $\|\sin \theta x+\cos \theta y\|^{\prime} \neq 0$, these mean that $\frac{\sin \theta x+\cos \theta y}{\|\sin \theta x+\cos \theta y\|^{\prime}}$ is in $A$ and is in the open arc of the complement of $A$ between $x$ and $y$ which is impossible.

When $\sim$ is $=$ the proof is in the similar way. The corresponding result for the case when $\sim$ stands for $\leq$ is also held in the similar method except that we must start from the minimal ellipse circumscribed $S$ instead.

Remark 2.4. In the similar method we could show that if $\theta_{1} \in(0, \pi)-\left\{\frac{\pi}{2}\right\}$ and $\theta_{2} \in$ $(-\pi, 0)-\left\{\frac{-\pi}{2}\right\}$ and

$$
\sin \left(2 \theta_{2}\right)\left\|\sin \theta_{1} x-\cos \theta_{1} y\right\|^{2}-\sin \left(2 \theta_{1}\right)\left\|\sin \theta_{2} x-\cos \theta_{2} y\right\|^{2} \sim \sin \left(2 \theta_{2}\right)-\sin \left(2 \theta_{1}\right)
$$

for every $x, y \in \mathscr{X}$, then $\mathscr{X}$ is an inner product space. Now a problem:

Problem. Can the requirements $\theta_{1} \in(0, \pi)-\left\{\frac{\pi}{2}\right\}$ and $\theta_{2} \in(-\pi, 0)-\left\{\frac{-\pi}{2}\right\}$ be eliminated and the case is still on going?

In [5] the authors introduce the notation of angle $A(x, y)$ between two vectors $x$ and $y$ of a normed space. In the sequel, with a slight change, we want to offer a new definition of angle between vectors in a normed space. In this definition, we enter a new parameter $\theta$ and observe that the new definition is identical to the previous one in a particular case.

Definition 2.5. Let $(X,\|\|$.$) be a real normed space and x, y \in X$ with $x, y \neq 0$. Define

$$
A_{\theta}(x, y)=\cos ^{-1}\left[\frac{1-\left\|\sin \theta \frac{x}{\|x\|}-\cos \theta \frac{y}{\|y\|}\right\|^{2}}{\sin (2 \theta)}\right]
$$

for some $\theta \neq \frac{k \pi}{2}$.

Note that in the particular case $\theta=\frac{\pi}{4}$, our definition and the definition noted earlier are identical.

Remark 2.6. The above definition is meaningful. In fact by the triangle inequality when $\sin (2 \theta) \geq 0$ we have

$$
1-\sin (2 \theta) \leq\left\|\sin \theta \frac{x}{\|x\|}-\cos \theta \frac{y}{\|y\|}\right\| \leq 1+\sin (2 \theta) .
$$

In the other case, the reverse inequalities are obtained. We note that when $\mathscr{X}$ is an inner product space with inner product $\langle.,$.$\rangle , then$

$$
A_{\theta}(x, y)=\cos ^{-1}\left[\frac{\langle x, y\rangle}{\|x\|\|y\|}\right] .
$$


$A_{\theta}(.,$.$) takes advantage many properties of A(.,$.$) mentioned in [5]. For instance using$ the argument in this paper, one can show that the angle construction is held for $A_{\theta}(.,$.$) .$ It asserts that for any two independent vectors $x$ and $y$ in $\mathscr{X}$ and $\alpha \in[0, \pi]$, there exists an $a \in \mathbb{R}$ such that $A_{\theta}(x, a x+y)=\alpha$. Also the other properties follow immediately from the definition which are listed below.

Lemma 2.7. Let $\theta \neq \frac{k \pi}{2}$. Then

i) $A_{\theta}(-x,-y)=A_{\theta}(x, y)$,

ii) $A_{\theta}(x, y)=A_{\theta}(\alpha x, \beta y)$ for all positive numbers $\alpha$ and $\beta$,

iii) $A_{\theta}(x, x)=0$,

iv) $A_{\theta}(x,-x)=A_{\theta}(-x, x)=\pi$.

However it does not satisfy some properties which are naturally expected. For example $A_{\theta}(x, y)=A_{\theta}(y, x)$ is not the case in general. To see this let $\mathscr{X}=\mathbb{R}^{2}$ with $\|\cdot\|_{\infty}$ and $x=(1,2), y=(2,3)$ and $\theta=\frac{\pi}{6}$. But obviously this equality holds in inner product spaces. In fact utilizing the following result of Lorch we show that It characterizes the inner product spaces.

Lemma 2.8. [11] A normed space $\mathscr{X}$ is an inner product space if and only if there exist a fixed constant $\gamma \neq 0,1$ such that $\|x\|=\|y\|$ implies $\|x+\gamma y\|=\|\gamma x+y\|$ for any $x$ and $y$ in $\mathscr{X}$.

Proposition 2.9. Let $\theta \neq \frac{k \pi}{4}$ and $A_{\theta}(x, y)=A_{\theta}(y, x)$ for all $x, y \in \mathscr{X}$. Then $\mathscr{X}$ is an inner product space.

Proof. $A_{\theta}(x, y)=A_{\theta}(y, x)$ implies that

$$
\left\|\sin \theta \frac{x}{\|x\|}-\cos \theta \frac{y}{\|y\|}\right\|=\left\|\sin \theta \frac{y}{\|y\|}-\cos \theta \frac{x}{\|x\|}\right\| .
$$

Hence

$$
\left\|\frac{x}{\|x\|}-\cot \theta \frac{y}{\|y\|}\right\|=\left\|\frac{y}{\|y\|}-\cot \theta \frac{x}{\|x\|}\right\|
$$

for every $x$ and $y$. Now what we want is concluded from Lemma 2.8.

Theorem 2.10. Let $\mathscr{X}$ be a real normed space and $\theta \neq \frac{k \pi}{2}$ such that $A_{\theta}(x, y)=A_{-\theta}(x, y)$ for any $x$ and $y$ in $\mathscr{X}$. Then $\mathscr{X}$ is an inner product space.

Proof. Let $x$ and $y$ be in $\mathscr{X}$. A routine computation shows that $A_{\theta}(x, y)=A_{-\theta}(x, y)$ is equivalent to

$$
\left\|\sin \theta \frac{x}{\|x\|}+\cos \theta \frac{y}{\|y\|}\right\|^{2}+\left\|\sin \theta \frac{x}{\|x\|}-\cos \theta \frac{y}{\|y\|}\right\|^{2}=2 .
$$

Now what we want is concluded from Theorem 2.3.

By this theorem and the remark after Theorem 2.3, it is easy to see that when $A_{\theta_{1}}(x, y)=$ $A_{\theta_{2}}(x, y)$ for any $x, y \in \mathscr{X}$ where $\theta_{1} \in(0, \pi)-\left\{\frac{\pi}{2}\right\}$ and $\theta_{2} \in(-\pi, 0)-\left\{\frac{-\pi}{2}\right\}$ then $\mathscr{X}$ is an inner product space.

In [5] the authors obtained several characterizations of inner product spaces based on the concept of angle between vectors $A(.,$.$) . Their proofs work good enough for A_{\theta}(.,$.$) .$

In what follows, we assume that $\sim$ is any one of $\geq, \leq$ or $=$ and $\theta \neq \frac{k \pi}{2}$.

Theorem 2.11. The following statements are equivalent;

i) $A_{\theta}(x, y)+A_{\theta}(-x, y) \sim \pi$ for all $0 \neq x, y \in \mathscr{X}$.

ii) $\left.A_{\theta}(x, a x+b y)+A_{\theta}(y, a x+b y) \sim A_{(} x, y\right)$ for all $x, y \in \mathscr{X}$ and all $a, b>0$.

iii) $A_{\theta}(x, y)+A_{\theta}(y-x, y)+A_{\theta}(x, x-y) \sim \pi$ for all independent vectors $x, y \in \mathscr{X}$.

iv) $A_{\theta}(y, y-x)+A_{\theta}(x, x-y) \sim A_{\theta}(-x, y)$ for all independent vectors $x, y \in \mathscr{X}$.

v) $\mathscr{X}$ is an inner product space. 
Proof. Obviously v) implies the other statements thus suffice it showing any one of i), ii), iii) and iv) implies v).

Let i) be held. Utilizing Theorem 2.3 it suffices to prove $\|\sin \theta x-\cos \theta y\|^{2}+\| \sin \theta x+$ $\cos \theta y \|^{2} \sim 2$ when $\|x\|=\|y\|=1$. Suppose that $A_{\theta}(x, y)+A_{\theta}(-x, y) \leq \pi$ for all $0 \neq x, y \in$ $X$ and $\sin (2 \theta) \leq 0 . x$ and $y$ so are assuming that have the norm 1 . From the definition $\|\sin \theta x-\cos \theta y\|=1-\sin (2 \theta) \cos A_{\theta}(x, y)$ and $\|\sin \theta x+\cos \theta y\|=1-\sin (2 \theta) \cos A_{\theta}(-x, y)$.

But by i) we have that $\cos A_{\theta}(-x, y) \geq-\cos A_{\theta}(x, y)$ which implies that $\| \sin \theta x-$ $\cos \theta y\left\|^{2}+\right\| \sin \theta x+\cos \theta y \|^{2} \geq 2$. In the case when $\sin (2 \theta) \geq 0$ we reach to $\| \sin \theta x-$ $\cos \theta y\left\|^{2}+\right\| \sin \theta x+\cos \theta y \|^{2} \leq 2$.

ii) $\rightarrow \mathrm{v}$ ) We prove i). Suppose $0 \neq x, y \in \mathscr{X}$. Without loss of generality we may assume that $x$ and $y$ are linearly independent vectors. Then for any positive real numbers $a$ and $b$ ii) shows that

$$
A_{\theta}(x, y)+A_{\theta}(b y-a x, y) \sim A_{\theta}(x, b y-a x) .
$$

But $A_{\theta}(x, b y-a x)=A_{\theta}\left(x, \frac{b}{a} y-x\right)$ and $A_{\theta}(b y-a x, y)=A_{\theta}\left(\frac{b}{a} y-x, y\right)$. Thus $\lim _{b \rightarrow 0} A_{\theta}(x, b y-$ $a x)=A_{\theta}(x,-x)=\pi$ and $\lim _{b \rightarrow 0} A_{\theta}(b y-a x, y)=A_{\theta}(-x, y)$ hence $A_{\theta}(x, y)+A_{\theta}(-x, y) \sim$ $\pi$.

iii) $\rightarrow$ v) Again we show i) is satisfied for independent vectors $x, y \in X$. By iii) and this fact that for positive numbers $\alpha$ and $\beta, A_{\theta}(\alpha x, \beta y)=A_{\theta}(x, y)$ we see that

$$
A_{\theta}(x, y)+A_{\theta}(y-n x, y)+A_{\theta}(x, n x-y) \sim \pi .
$$

Due to Remark 2.6 and the continuity we have $\lim _{n \rightarrow \infty} A_{\theta}(y-n x, y)=A_{\theta}(-x, y)$ and $\lim _{n \rightarrow \infty} A_{\theta}(x, n x-y)=A_{\theta}(x, x)=0$, so it follows that $A_{\theta}(x, y)+A_{\theta}(-x, y) \sim \pi$.

iv) $\rightarrow$ v) It is easy to see that for positive numbers $a$ and $b$,

$$
\begin{aligned}
A_{\theta}(x, a x+b y)+A_{\theta}(y, a x+b y) & \sim A_{\theta}(a x, a x+b y)+A_{\theta}(-b y,-a x-b y) \\
& \sim A_{\theta}(a x, b y)=A_{\theta}(x, y) .
\end{aligned}
$$

Now the result is concluded from ii).

The definition 2.5 leads to an associated definition of orthogonality.

Definition 2.12. Let $x, y \in \mathscr{X}$. We say that $x$ is $\theta$-orthogonal to $y$ for some $\theta \neq \frac{k \pi}{2}$, denoted by $x \perp_{\theta} y$, if $x=0$ or $y=0$ or if $x \neq 0$ and $y \neq 0$ and

$$
\left\|\sin \theta \frac{x}{\|x\|}-\cos \theta \frac{y}{\|y\|}\right\|=1 \text {. }
$$

Equivalently, $x \perp_{\theta} y$ if and only if $\|\sin \theta x\| y\|-\cos \theta y\| y\|\|=\|x\|\|y\|$.

This orthogonality has the following properties as the orthogonality introduced in [5].

i) $0 \perp_{\theta} x$ and $x \perp_{\theta} 0$ for all $x \in \mathscr{X}$,

ii) $x \perp_{\theta} y$ implies $\alpha x \perp_{\theta} \beta y$ for positive numbers $\alpha$ and $\beta$,

iii) for all $x, y \in \mathscr{X}$ there exists $\alpha \in \mathbb{R}$ such that $x \perp \alpha x+y$,

iv) $\theta$-orthogonality is homogeneous if and only if $x \perp_{\theta} y$ implies $x \perp_{\theta}-y$.

Many of the issues raised in [5] are presented here as well. For example neither orthogonality nor $\theta$-orthogonality are additive. In [5] it was shown that the additivity of the orthogonality may not characterize inner product spaces even with the additional assumption of strict convexity of the spaces. In the following we try to approach this problem. For this aim we use a result of Lorch in [11]. It reads as follows:

Lemma 2.13. A normed space $\mathscr{X}$ is an inner product space if and only if for some $\xi \neq 0,1$ and $-1,\|x-y\|=\|x+y\|$ implies $\|x-\xi y\|=\|x+\xi y\|$ for any $x, y \in \mathscr{X}$.

Theorem 2.14. Let $X$ be a normed space. Then the following statement are equivalent:

i) There exist two distinct numbers $\theta_{1} \neq \frac{\pi}{4}$ and $\theta_{2}$ in $\left(0, \frac{\pi}{2}\right)$ such that $\|x-y\|=\|x+y\|$ implies $x \perp_{\theta_{1}} y$ and $x \perp_{\theta_{2}} y$ for all $x$ and $y$. 
ii) For some $\theta \neq \frac{k \pi}{2},\|x-y\|=\|x+y\|$ if and only if $x \perp_{\theta} y$ for any $x$ and $y$ in $\mathscr{X}$.

iii) $\mathscr{X}$ is an inner product space.

Proof. Obviously iii) implies the other statements thus suffice it showing any one of i), ii) implies iii).

i) $\rightarrow$ iii)Let $x$ and $y$ be two non-zero vectors in $\mathscr{X}$ such that $\|x-y\|=\|x+y\|$. By i) we have that $x \perp_{\theta_{1}} y$ and $x \perp_{\theta_{1}}-y$, which is

$$
\left\|\sin \theta_{1} \frac{x}{\|x\|}-\cos \theta_{1} \frac{y}{\|y\|}\right\|=1=\left\|\sin \theta_{1} \frac{x}{\|x\|}+\cos \theta_{1} \frac{y}{\|y\|}\right\| .
$$

This implies that

$$
\left\|\frac{x}{\|x\|}-\cot \theta_{1} \frac{y}{\|y\|}\right\|=\left\|\frac{x}{\|x\|}+\cot \theta_{1} \frac{y}{\|y\|}\right\| .
$$

Thus again by i) we have that

$$
\left\|\frac{x}{\|x\|}-\frac{\cot \theta_{2}}{\cot \theta_{1}} \frac{y}{\|y\|}\right\|=\left\|\frac{x}{\|x\|}+\frac{\cot \theta_{2}}{\cot \theta_{1}} \frac{y}{\|y\|}\right\| .
$$

By repeating this process we see that

$$
\left\|\frac{x}{\|x\|}-\left(\frac{\cot \theta_{2}}{\cot \theta_{1}}\right)^{n} \frac{y}{\|y\|}\right\|=\left\|\frac{x}{\|x\|}+\left(\frac{\cot \theta_{2}}{\cot \theta_{1}}\right)^{n} \frac{y}{\|y\|}\right\|
$$

for all integer $n$. By our assumption we know that $\frac{\cot \theta_{2}}{\cot \theta_{1}}$ is not any one of 0,1 or -1 . Now utilizing the Lemmata 2, 3 and 4 of [11] we conclude that

$$
\left\|\frac{x}{\|x\|}-\xi \frac{y}{\|y\|}\right\|=\left\|\frac{x}{\|x\|}+\xi \frac{y}{\|y\|}\right\|
$$

for every real number $\xi$ and this implies, in turn, that $\|x-\xi y\|=\|x+\xi y\|$ for any real number $\xi$. This obviously means that $\mathscr{X}$ is an inner product space.

ii) $\rightarrow$ iii) Let $x$ and $y$ be in $\mathscr{X}$ such that $\|x-y\|=\|x+y\|$. Thus by our assumption in ii) $x \perp_{\theta} y$. Thus $x \perp_{\theta} \xi y$ for any positive number $\xi$ and this implies that $\|x-\xi y\|=\|x+\xi y\|$. Thus the result is concluded from the Lemma 2.13.

\section{References}

[1] D. Amir, Characterizations of Inner Product Spaces, Operator theory: Advances and applications,20, Birkhäuser Verlag, Besel, 1986.

[2] F. Dadipour and M.S. Moslehian, A characterization of inner product spaces Related to the p-angular distance, J. Math. Anal. App. 371 (11), 677-681, 2010.

[3] M.M. Day, Some characterizations of inner product spaces, Trans. Amer. Math. Soc. 62, 320-337, 1947.

[4] M.M. Day, Normed Linear spaces, 3th edition, Springer, New York, 1973.

[5] C.R. Diminnie, E.Z. Andalafte and R.W. Freese, Angles in normed linear spaces and a characterization of real inner product spaces, Math. Nachr. 129, 197-204, 1986.

[6] C.R. Diminnie, E.Z. Andalafte and R.W. Freese, Angle bisectors in normed linear spaces, Math. Nachr. 131, 167-173, 1987.

[7] M. Frećhet, Sur la definition axiomatique d'une classe d'espaces vectoriels distanciés applicables vectoriellement sur l'espace de Hilbert, Ann. of Math. (2), 36 (3), 705-718, 1986.

[8] R.C. James, Inner product in normed linear spaces, Bull. Amer. Math. Soc. 53, 559$566,1947$.

[9] R.C. James, Orthogonality and linear functionals in normed linear spaces, Trans. Amer. Math. Soc. 61, 265-292, 1947.

[10] P. Jordan and J. von Neumann, On inner product in linear metric spaces, Ann. of Math. (2), 36 (3), 719-723, 1935. 
[11] E.R. Lorch, On certain implications which characterize Hilbert space, Ann. of Math. (2), 49 (2), 523-532, 1948. 